\title{
Familial Pelger-Huet Anomaly
}

\author{
Samir S. Shah • Rupali S. Parikh · Lakshmi P. Vaswani • \\ Rajeshree Divkar
}

Received: 25 September 2014/ Accepted: 17 January 2015/Published online: 25 January 2015

(C) Indian Society of Haematology \& Transfusion Medicine 2015

\begin{abstract}
Pelger-Huet anomaly is usually autosomal dominant, although it is likely that new mutations are common. This condition is characterized by granulocytes that are either bilobed or completely unsegmented. Here is a report of a 46 year old Indian lady who presented with fever to the hospital and on evaluation, her peripheral blood smear showed extreme hypolobation of granulocytes. Along with normal appearing neutrophils there were many neutrophils with bilobed and single monolobated nuclei which accounted for $82 \%$ of the neutrophils. After identifying these neutrophilic abnormalities which were suggestive of Pelgeroid changes, the other family members were also screened and were found to be having similar morphologic abnormalities in granulocytes. As these changes were evident in granulocytic leucocytes of the patient as well as her mother, both her sisters and her son, with exception of her brother, the diagnosis of familial Pelger-Huet Anomaly was considered in this case.
\end{abstract}

Keywords Familial · Pelger-Huet · Anomaly · Bilobed · Granulocytic leucocytes $\cdot$ Pince-Nez

\section{Introduction}

Pelger-Huet anomaly is a benign, dominantly inherited defect of terminal neutrophil differentiation secondary to

Communicated by Haraprasad Pati.

Electronic supplementary material The online version of this article (doi:10.1007/s12288-015-0508-3) contains supplementary material, which is available to authorized users.

S. S. Shah $(\bowtie) \cdot$ R. S. Parikh · L. P. Vaswani · R. Divkar Laboratory, Bhatia Hospital, 2nd Floor, Tukaram Javji Road, Tardeo, Mumbai 400007, India

e-mail: dr_samirshah@hotmail.com mutations in the Lamin B Receptor gene (LBR gene). This gene maps on 1q41-q43 [1].

It is characterized by distinctive shapes of nuclei of granulocytes, a reduced number of nuclear segments and coarseness of chromatin of nuclei of neutrophils, lymphocytes and monocytes. The unilobed form of the PelgerHuet cells is occasionally called Stodmeister forms. These cells can be predominant cell type in exceedingly rare homozygous forms of Pelger-Huet anomaly. Homozygous patients may have varying degrees of developmental delay, epilepsy and skeletal abnormalities [1].

The incidence of heterozygous state of Pelger-Huet anomaly was reported to be one in six thousand, while the homozygous states are very rare and less than ten cases have been reported [2].

\section{Case Report}

A 46 year old Indian lady came to the hospital for blood work up for fever. Examination of her blood smear was remarkable for neutrophils with predominance of bilobed, spectacle shaped nuclei, an appearance often described as Pince-Nez.

Her WBC count was $3.55 \times 10^{9} / \mathrm{L}$, platelet count was $176 \times 10^{9} / \mathrm{L}$, hematocrit $33.1 \%$, MCV $75.2 \mathrm{fl}$, hemoglobin $10.8 \mathrm{~g} / \mathrm{dl}$.

The peripheral smear showed $67 \%$ neutrophils, $22 \%$ lymphocytes, $2 \%$ eosinophils and $9 \%$ monocytes. The absolute neutrophil count was 2379 cells/cumm. Out of the $67 \%$ of neutrophils, normal appearing neutrophils with three lobes were $9 \%$, while cells with bilobed and single monolobated nuclei accounted for $82 \%$ and intermediate forms were $9 \%$. 
After identifying these neutrophilic abnormalities which were suggestive of Pelgeroid changes, the other family members were screened and were found to be having similar morphologic abnormalities in granulocytes. No history of frequent or increased incidences of infections was elicited from the patient and her family members (Table 1; Figs. 1, 2, 3).

Pelger-Huet anomaly was evident in granulocytic leucocytes of the patient, her mother, both her sisters and her son. However her brother did not show this anomaly.

\section{Discussion}

The first observation of abnormal hyposegmented granulocytes was made by Huet in 1928. A year later, Pelger reported another case. Such a morphological leukocyte anomaly later became known as Pelger-Huet anomaly [2]. These cells are typically found only in manual review of peripheral blood smears because automated hematology analyzers do not detect the neutrophils in the patients with Pelger-Huet anomaly as abnormal [1].

Pelger-Huet anomaly is characterized by granulocytes that are bilobed or completely unsegmented. The chromatin is mature with heavy condensation [1]. The homozygous form of Pelger-Huet anomaly is characterized by extreme hypolobation of peripheral blood granulocyte nuclei. Almost all nuclei are bilobed or have only a single oval or rod shaped nucleus [1]. These are not normal band forms of neutrophils but rather functionally normal neutrophils and this should not be confused with left shifted maturation of neutrophils [2].

The morphological mimics of Pelger-Huet anomaly are more common than the true disorder. The changes of hypolobation can be seen primarily as a reactive condition in association with medications or drugs like mycophenolate or valproate, or in cases of myelodysplastic syndrome or other related myeloid disorders. When this hypolobation is seen under these circumstances, it is referred to as PseudoPelger Huet or Pelgeroid changes. [1].

In case of myelodysplasia or myeloid neoplasm, the hypolobation is often accompanied by hypogranulation of neutrophils as well as other dysplastic changes seen in

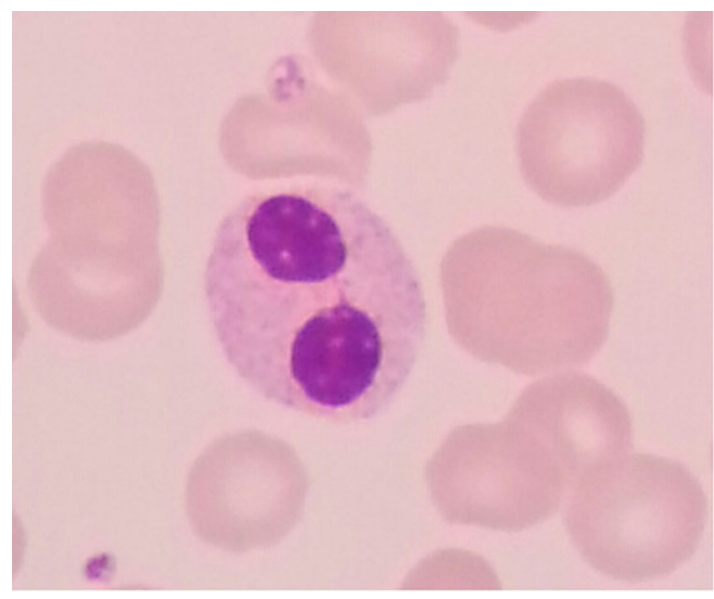

Fig. 1 Pelger Huet cell with bilobed nucleus

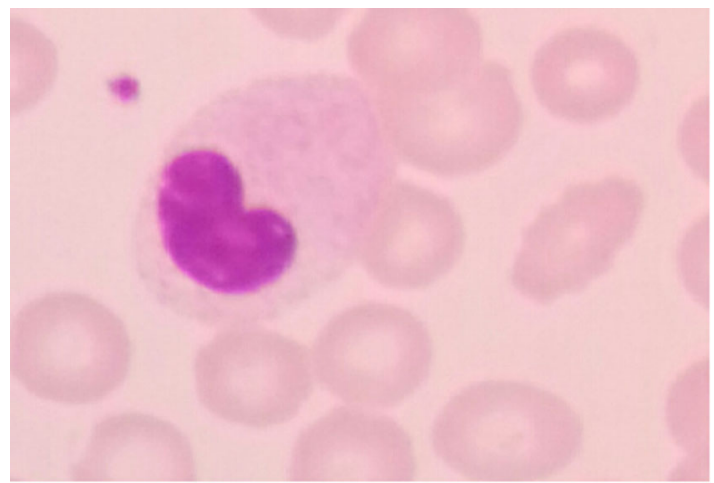

Fig. 2 Pelger Huet cell-short band cell

erythroid, platelet/megakaryocytic cells. In particular, isochrome $17 \mathrm{q}$ or $17 \mathrm{p}$ minus syndromes are the cytogenetic abnormalities associated with these changes [1]. The Pelgeroid cells which are seen in the acquired form of myelodysplasia are considered to be manifestations of apoptosis [2].

In both reactive conditions and dysplastic type changes, only a subset of neutrophils is usually affected, in contrast to the entire population of cells in Pelger-Huet anomaly [1].

In infection associated cases, toxic changes of granulocytes may also be seen [2]. Identification of drug related
Table 1 Complete blood counts of the patient and her family members

\begin{tabular}{llllll}
\hline & WBC $\left(10^{9} / \mathrm{L}\right)$ & Platelets $\left(10^{9} / \mathrm{L}\right)$ & Hematocrit $(\%)$ & MCV $(\mathrm{fL})$ & Hemoglobin $(\mathrm{g} / \mathrm{dL})$ \\
\hline Patient & 3.55 & 176 & 33.1 & 75.2 & 10.8 \\
Sister 1 & 8.64 & 310 & 35.2 & 80.7 & 11.6 \\
Sister 2 & 7.79 & 280 & 36.1 & 84.3 & 11.3 \\
Mother & 7.80 & 265 & 30.2 & 86.8 & 9.6 \\
Son & 5.79 & 176 & 40.2 & 86.1 & 13.8 \\
Brother & 5.84 & 347 & 44.4 & 88.3 & 15.4 \\
\hline
\end{tabular}




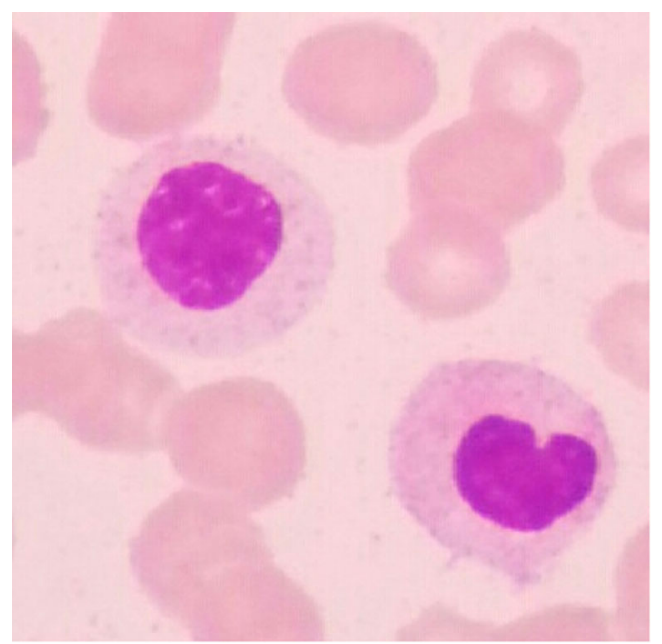

Fig. 3 Pelger Huet cells with unsegmented nucleus and short band cell

cases require knowledge of clinical history and high index of suspicion [1].

It has been assumed that Pelger-Huet anomaly has no clinical effects. Experiments done on Pelger-Huet granulocytes regarding their biomedical, phagocytic, metabolic and bactericidal activities as well as their motility and responses to chemotactic stimuli are all equivalent to normal granulocytes [3].

However a higher incidence of local infection was reported and that might be limited to morphological changes and impaired ability of affected granulocytes to migrate through small blood vessels [4].

But, in our case neither the patient nor any of her relatives showed any symptoms associated with granulocyte malfunction such as increased incidence of infections.

In recent times, the Ham's Classification System has been used to differentiate between the different types of granulocytes in a case of suspected Pelger-Huet anomaly.

By this system, neutrophilic leukocytes stained with Leishman stain may be divided into three types: type A (normal), type B (intermediate) and type C (typical PelgerHuet).
Table 3 Percentage of type A, type B and Type C cells according to Ham's classification

\begin{tabular}{llll}
\hline & $\begin{array}{l}\text { Type A cells } \\
(\%)\end{array}$ & $\begin{array}{l}\text { Type B cells } \\
(\%)\end{array}$ & $\begin{array}{l}\text { Type C cells } \\
(\%)\end{array}$ \\
\hline Patient & 9 & 9 & 82 \\
Sister 1 & 18 & 15 & 67 \\
Sister 2 & 30 & 18 & 52 \\
Mother & 22 & 18 & 60 \\
Son & 19 & 11 & 70 \\
Brother & 100 & - & - \\
\hline
\end{tabular}

Type C cells characteristically have fewer nuclear lobes. Cells with unsegmented nuclei predominate and those with more than two nuclear lobes are rare. The nuclear chromatin of these cells is very basophilic and densely clumped, giving a pitted appearance. The nuclear outline is smooth. The nuclei of unsegmented cells are round, ovoid or short thick, plump rods. Bilobed cells are either symmetrical or asymmetrical. The most characteristic and diagnostic cell is the so called Pince-Nez form, in which the two lobes of the nucleus are round symmetrical and joined by a thin chromatin strand.

The type B cells exhibit these properties to a lesser extent and tend toward the appearance of normal cells [5].

The tables below show the differential counts of the peripheral blood smear of the patient and the members of her family. The classification of granulocytes is done in accordance with Ham's Classification (Tables 2, 3).

\section{Conclusion}

The unique morphological features identified on well-prepared and well-stained peripheral blood smears and the review of patient's clinical and family history are needed to separate Pelger Huet Anomaly from Pseudo-Pelger Huet Anomaly. Family screening for the disorder is recommended to identify other affected family members.
Table 2 Differential count performed on Leishman stained smears of the patient and her other family members

\begin{tabular}{llllll}
\hline & $\begin{array}{l}\text { Neutrophils } \\
(\%)\end{array}$ & $\begin{array}{l}\text { Lymphocytes } \\
(\%)\end{array}$ & $\begin{array}{l}\text { Eosinophils } \\
(\%)\end{array}$ & $\begin{array}{l}\text { Monocytes } \\
(\%)\end{array}$ & $\begin{array}{l}\text { Basophils } \\
(\%)\end{array}$ \\
\hline Patient & 67 & 22 & 2 & 9 & 0 \\
Sister 1 & 75 & 17 & 3 & 5 & 0 \\
Sister 2 & 74 & 20 & 1 & 5 & 0 \\
Mother & 51 & 40 & 4 & 5 & 0 \\
Son & 47 & 44 & 4 & 5 & 0 \\
Brother & 63 & 25 & 5 & 7 & 0 \\
\hline
\end{tabular}




\section{References}

1. O’Malley D, Hsi E (2007) Non neoplastic morphologic abnormalities of white blood cells and macrophages. In: Goldblum J (ed) Hematopathology, a volume in the series foundations in diagnostic pathology, 1st edn. Elsevier, Philadelphia, pp 55-66

2. Al-Qaddomi A, Bassma H, Wraikat A et al (2006) Pelger -Huet anomaly first report from Jordan. A case report. JRMS 13(2): $54-56$
3. Yamasoma Y, Fujji T, Tsuchiatori K (1953) The Pelger-Huet familial anomaly of leukocytes. Blood 8:370-374

4. Johnson CA, Bass D, Trilloy A et al (1980) Functional and metabolic studies of polymorphonuclear leucocytes in the congenital Pelger -Huet anomaly. Blood 55(3):466-469

5. Rosse W, Gurney C (1959) The Pelger-Huet anomaly in three families and its use in determining the disappearance of transfused neutrophils from the peripheral blood. Blood 14:170-186 